\title{
UNA APROXIMACIÓN AL ESTUDIO DE LA CONCENTRACIÓN INDUSTRIAL
}

POR

M. M. MAYORAL

\section{Introducción}

El estudio de la localización y concentración industrial como objetivo de la Geografía Industrial ha acercado a la Geografía y Economía más que cualquier otra disciplina de la investigación geográfica. Así, es importante destacar el papel que juega el territorio en el desarrollo de la economía y prueba de ello es que desde sus orígenes la Geografía Industrial está interesada no sólo en la distribución de la actividad manufacturera sino también en los diferentes factores que influyen en la toma de decisiones sobre la localización y organización espacial, es decir, factores que favorecen la concentración industrial: infraestructura viaria, mano de obra barata, proximidad de materias primas, precio del suelo, proximidad a la aglomeración de población, disponibilidad de suelo industrial así como la atracción que puedan ejercer empresas ya ubicadas que junto con la instalación de los centros de control y adopción de decisiones explican la tendencia de la actividad económica.

El marco de referencia para aproximarnos al estudio de la concentración es el mercado, de esta forma, definimos concentración como el grado por el cual la producción se encuentra en manos de un reducido número de empresas en un mercado o industria concreta.

M. M. Mayoral. Departamento de Metodología de la Investigación. Escuela Universitaria de Estadística Universidad Complutense de Madrid. 
Por ello, cualquier ratio que se considere para cuantificar la concentración debe reflejar el grado de oligopolio existente en un mercado, de forma que al combinar las cuotas de mercado de un grupo de 3, 4 ó 5 empresas muy grandes, muestre el poder ejercido por un grupo interdependiente. Ratios altos deben de indicar que unas pocas empresas dominan el mercado y por tanto será más factible la colusión, o sea la cooperación, entre ellas. La elevada concentración junto con las condiciones estables del mercado así como la homogeneidad en oferta y demanda contribuirá a favorecer la coordinación oligopolística y ello dará lugar a precios más altos.

Es importante señalar que a partir de los años setenta, existe una clara tendencia a conferir mayor importancia a la medición de las cuotas individuales de cada empresa en el mercado que a los índices de concentración. A pesar de ello éstos siguen siendo de gran utilidad en la descripción estadística de diversas industrias.

En este trabajo, se abordan algunas cuestiones relacionadas con la concentración industrial. Al medir la concentración industrial o de mercado resulta necesario conocer el número de participantes que hay en el mismo así como el tamaño relativo de cada uno de ellos, abordando en el apartado 2 algunas cuestiones relacionadas con la problemática que surge en relación con la concentración industrial.

En el apartado 3 se describen algunas de las medidas que han sido utilizadas en diferentes estudios pasando por el Ratio o Coeficiente de Concentración así como por los índices basados en la participación de todas las empresas. Finalmente, en el último apartado, se abordan las medidas de concentración desde el enfoque de la Teoría de la Información, menos habituales, poniendo de manifiesto la verificación de aquellas propiedades que parece razonable verifique un «buen» índice de concentración industrial.

\section{Propiedades deseables de una medida de concentración}

Los índices que se han venido utilizando en diferentes estudios sobre concentración industrial tienen como finalidad dar una aproximación de la estructura del mercado a través principalmente de los tamaños de las unidades que participan en el mismo. Hay que ser conscientes de que elegir cómo cuantificar el tamaño lleva consigo 
una gran dificultad debido a la complejidad de esa realidad que intentamos abordar. Esta complejidad se traduce en cómo elaboramos un índice que esté definido sobre una variable representativa del tamaño de las empresas, no existiendo criterio unánime en la selección de esa variable. Cuestión ésta de especial importancia y que en muchos casos no se tiene en cuenta, optándose por la elección más o menos arbitraria de dicha variable.

Entre las variables más apropiadas para medir el tamaño de las empresas se han señalado: ventas, valor añadido, potencia eléctrica instalada, empleo y activos, entre otras; lógicamente la utilización de una u otra va a repercutir en el valor de la medida. También habrá que tener en cuenta los inconvenientes que conllevan la utilización de dichas variables como señala González, J.L. (1988). No obstante Bayle, D. y Boyle, S.E. (1971), probaron que existe una fuerte correlación entre los diferentes grados de concentración utilizando distintas variables, por lo que la elección de una u otra no tendrá gran importancia excepto si queremos realizar comparaciones (Eraso, L. y García, C. (1990)). Por todo ello, a la hora de optar por una de ellas se tendrá en cuenta la disponibilidad de información, lo que lleva generalmente a la consideración del empleo como variable más comunmente utilizada para la evaluación de la concentración. Parece lógico pensar que en muchas ocasiones ésta no será la característica que mejor refleje la capacidad productiva de una industria debido fundamentalmente a las diferencias de los métodos y técnicas de producción en las distintas empresas, pero es para la que se dispone de información más fiable.

Una vez resuelto el primer problema relacionado con la selección de la variable a estudiar, un segundo problema que surge es el asociado a la definición correcta del mercado, ya que mientras algunos autores se centran en industrias definidas a cuatro dígitos otros consideran que la definición de las industrias a cinco dígitos es la más correcta. Ahora bien será necesario recurrir a la desagregación a cinco dígitos cuando se estima una cierta importancia en función de la variable a analizar y cuando realmente se observa una especialización. Por último una tercer cuestión que surge está referida a la selección del índice que mejor refleje el nivel de concentración existente.

En general, podemos diferenciar dos tipos de índices de concentración industrial: 
a) Los índices parciales que sólo reflejan una parte, normalmente el tramo superior correspondiente a las empresas de tamaño más elevado, de la distribución de los tamaños de las empresas que integran el mercado.

b) En contraposición a los primeros hay que señalar los índices completos, éstos consideran el conjunto de la distribución de los tamaños.

Todos estos índices cuantifican la participación ponderada de las empresas en el mercado, frente a las medidas de desigualdad de la concentración que miden el grado de dispersión a partir de un determinado valor medio. Estas medidas, como pone de manifiesto Marfels, C. (1971), se obtienen generalmente a partir de medidas de concentración.

Estos índices deben cumplir unos criterios que hagan aconsejable su utilización. Entre los criterios más elementales, como señala Clarke, R. (1993), hay que destacar:

1. Que sea fácil de comprender y calcular.

2. Debe ser independiente del tamaño, o, equivalentemente, el índice debe depender de la cuota de mercado.

3. Debe oscilar dentro de unos límites, 0 en el caso competitivo de muchas empresas con tamaños similares y 1 en el caso de monopolio. Esta propiedad no es determinante pero nos permite establecer comparaciones.

No obstante, un índice puede carecer de alguno de estos criterios y seguir siendo útil, dependerá principalmente de los objetivos que se persiguen. La elección de un buen indicador que nos permita cuantificar en la práctica el grado de concentración es una cuestión importante. Por ello, parece razonable seleccionar un índice que posea una serie de propiedades que intuitivamente resulta lógico exigir a una medida de concentración. Algunos autores como Hall, M. y Tideman, N. (1967) y Hannah, L. y Kay, J.A. (1977) se preocuparon por estudiar ciertos conjuntos de propiedades que, en principio, deberá satisfacer un buen índice de concentración industrial. Así pues, las axiomáticas propuestas por estos autores constituyen conjuntos de propiedades deseables, completamente aceptadas, que permiten caracterizar a dichas medidas y que son utilizadas para seleccionar un índice como una buena medida de concentración si éste cumple dichas propiedades. 
En cuanto a las propiedades que recoge la axiomática de Hall, M. y Tideman, N. (1967) estas son:

1) Un índice de concentración debe ser una medida adimensional. Es decir, una medida de concentración debe establecer un orden completo en el conjunto de industrias. Así, dadas dos distribuciones de mercado cualesquiera debemos ser capaces de determinar si una conlleva una mayor concentración que la otra o si por el contrario están igualmente concentradas.

2) La concentración de una industria debe ser independiente del tamaño de la misma, esto es, la importancia relativa de cada firma viene recogida por su participación en el mercado.

3) La concentración debe aumentar si la participación de una firma aumenta a expensas de otra firma más pequeña. Propiedad ésta conocida con el nombre de principio de transferencia de DaltonPigou (Pigou, A.C. (1912), Dalton, H. (1920)) y que refleja la influencia que tiene un cambio en la desigualdad de los tamaños relativos de las empresas, permaneciendo inalterado su número.

4) Si todas las firmas se dividen en $\mathrm{k}$ partes iguales, entonces el'i ndice debe reducirse en la proporción $(1 / \mathrm{k})$, o equivalentemente, aplicado a la fusión de empresas, si se unen $\mathrm{k}$ firmas de igual tamaño entonces la concentración aumenta en esa misma proporción.

5) Si hay $\mathrm{N}$ firmas de igual tamaño, la concentración tendrá que decrecer en función de N. Esto es, cuantas más empresas se repartan el mercado mayor es el nivel de competencia entre ellas y, por tanto menor será el grado de concentración.

6) Una medida de concentración deberá oscilar entre 0 y 1.

La otra axiomática debida a Hannah, L. y Kay, J.A. (1977), en la cual se adopta un enfoque puramente económico del problema, establece el siguiente conjunto de propiedades:

1) Situadas las empresas en orden decreciente de tamaño, un incremento en la participación acumulada de la j-ésima firma, para cualquier j, implica un incremento en la concentración.

2) Se debe verificar el principio de transferencia.

3) La entrada de firmas en la parte inferior del ranking debe reducir la concentración.

4) La fusión de empresas debe aumentar la concentración.

5) Una diferenciación aleatoria de los productos por parte de los consumidores deberá reducir la concentración. 
6) Cuanto menor sea la participación de una nueva firma, menor será el efecto sobre el grado de concentración.

7) La influencia de factores aleatorios sobre el crecimiento de las empresas debe aumentar la concentración.

Todas estas propiedades, que evidentemente están interrelacionadas como ponen de manifiesto entre otros Río, M.J. y Pérez, R. (1988), delimitan el conjunto de posibles indicadores para medir la concentración industrial, simplificando, en parte, la elección de un índice adecuado pero no la resuelven por completo, pues, según veremos posteriormente existen diferentes índices que cumplen todas estas propiedades. A pesar de ello, constituyen un punto de referencia útil. Un amplio estudio sobre el significado y contenido normativo de todos estos axiomas puede verse en Curry, B. y George, K.D. (1983).

Otros autores que plantean conjuntos de propiedades razonables que permitan seleccionar medidas adecuadas de concentración son Egghe, L. y Rousseau, R. (1990, 1991).

\section{Medición de la concentración}

Desde que surge interés por el estudio de la concentración son numerosos los índices que han ido apareciendo en la literatura para tratar de cuantificar este fenómeno. A describir algunos de los índices de uso más frecuente, a analizar el por qué de su utilización, así como a considerar cuáles son sus defectos se dedica este apartado.

A partir de ahora supondremos un sector industrial integrado por $\mathrm{N}$ establecimientos o empresas con tamaños $x_{i}(\mathrm{i}=1, \ldots, \mathrm{N})$ clasificados de mayor a menor. Si definimos el tamaño del sector industrial como $x=\sum_{i=1}^{N} x_{i}$, la participación (tamaño relativo) del establecimiento o empresa i-ésima será $p^{i}=x^{i} / x$.

El índice más utilizado, en este contexto, es el Ratio o Coeficiente de Concentración que hace referencia al porcentaje de participación que representan las $\mathrm{K}$ empresas de mayor tamaño. Así, ordenadas éstas de mayor a menor de acuerdo a su participación $p_{i}$ dentro del sector, el índice viene dado por:

$$
-188-
$$




$$
C R_{K}=\sum_{i=1}^{K} p_{i}
$$

y presenta la gran ventaja de ser uno de los más sencillos que se pueden emplear en la cuantificación de la concentración industrial por lo que se encuentra ampliamente difundido. Este índice puede ser aplicado a otros grupos de empresas, no necesariamente las $\mathrm{K}$ mayores, como pone de manifiesto Miller, R.A. (1971). A pesar de todo, se han efectuado diferentes críticas al mismo entre las que se encuentra la que hace referencia a la selección arbitraria de $\mathrm{K}$, es decir, del número de empresas a tener en cuenta en la elaboración del índice ya que al representar sólo una parte de la distribución de los tamaños de las empresas integrantes del mercado, será invariante respecto al número de empresas así como a la distribución de los tamaños relativos del tramo considerado. Una transferencia de ventas o una fusión puede no producir un cambio en el ratio de concentración, como ocurre por ejemplo cuando las $\mathrm{K}$ mayores empresas no se ven afectadas. Por tanto, este índice no presenta un adecuado comportamiento con respecto a la axiomática de Hannah-Kay.

En ocasiones resulta interesante, partiendo del grado de concentración observado, encontrar el número de empresas que se repartirían el mercado equitativamente, manteniendo el mismo nivel de concentración. La comparación de este número, denominado Número Equivalente (Horowitz, I. (1970)), y el realmente observado nos permitirá extraer algunas conclusiones sobre la estructura del mercado.

Otro índice muy simple es el Recíproco del Número de Empresas, $1 / \mathrm{N}$. En este índice se observa la mayoría de las propiedades que hemos señalado anteriormente, a excepción de la que hace referencia a la transferencia de ventas que no lleva consigo ningún cambio en este índice.

Entre los índices basados en la participación de todas las empresas del sector, y no en una parte de ellas como ocurre con el ratio de concentración, cabe señalar:

a) Indice de Hirschman-Herfindahl: Este índice, propuesto por Hirschman, A.O. (1945, 1964) y Herfindahl, O.C. (1950) y estudiado entre otros por Stigler, G.J. (1968) y Aguiló, E. (1979), ha sido utilizado principalmente por los economistas industriales. El índice viene dado por: 


$$
H=\sum_{i=1}^{N} p_{i}^{2}
$$

Al estar los $p_{i}$, tamaños relativos, elevados al cuadrado, este índice da más importancia a las empresas de mayor tamaño y depende no sólo de la desigualdad en éstas sino también del número de empresas. Además, puede tomar un valor máximo de 1, en el caso de monopolio y un valor próximo a 0 , valor mínimo, siempre que exista gran número de empresas de igual tamaño. También hay que tener en cuenta que el recíproco de $\mathrm{H}$ es su número equivalente; es decir, valor que corresponde al número de empresas de igual tamaño que darían lugar a ese mismo valor de $\mathrm{H}$.

b) Indice de Hannah-Kay: Este índice, introducido por Hannah, L. y Kay, J. A. (1977), viene dado por:

$$
H K=\left(\sum_{i=1}^{K} p_{i}^{\alpha}\right)^{1 /(1-\alpha)}, \alpha y \quad \alpha \neq 1
$$

donde $\alpha$ es un parámetro arbitrario de elasticidad. Este índice es similar al analizado anteriormente en el caso $\alpha=2$. Además de cumplir, al igual que el anterior, los criterios generales recogidos en las dos axiomáticas consideradas en el apartado anterior, como puede verse en el trabajo de Curry,B. y George, K.D. (1983) en el que analizan el comportamiento de éstas y otras medidas ante las caracterizaciones mencionadas, da un mayor o menor peso a las empresas más grandes a través de los distintos incrementos de $\alpha$. Este índice coincide con su número equivalente, por lo que puede ser interpretado como una medida inversa de concentración.

c) Indice de Hall-Tideman: Este índice viene dado por:

$$
H K=\left(\frac{1}{2}\right)\left(\sum_{i=1}^{N} i p_{i}^{\alpha}\right)^{-1}-1
$$

y con él se pondera el peso de cada una de las firmas por el lugar que ocupa en el ranking. Este índice fue introducido y ampliamente estudiado por Hall, M. y Tideman, N. (1967), analizando su buen comportamiento frente a la axiomática planteada por ellos mismos. Otros estudios interesantes han sido realizados por Bayle, D. y Boyle, S.E. (1971) quienes comparan este índice con CR y H. 
d) Indice de concentración global: Propuesto por Horvath, J. (1970) asigna una alta ponderación a la empresa de mayor tamaño. En cuanto a las restantes disminuye la ponderación de las empresas mayores con respecto a las más pequeñas en relación a otros índices como por ejemplo al de Herfindahl. Este índice viene dado en los siguientes términos:

$$
C C I=p_{1}-\left(\sum_{i=2}^{N} p_{i}^{2}\right)\left(2-p_{i}\right) .
$$

e) Indice multiplicativamente modificado de Cournot: Este índice propuesto por Hause, J.C. (1977) viene dado en términos del índice de Herfindahl de la siguiente forma:

$$
H M(\alpha)=\sum_{\mathrm{i}=1}^{N} p_{i}\left(2-\left(p_{1}\left(H-p_{i}^{2}\right)\right)^{\alpha}\right), \alpha \geqslant 0.15
$$

Un estudio comparativo de este índice con $\mathrm{H}$ y $\mathrm{CR}$ ha sido realizado por Aguiló, E. (1979), quien calcula estos índices para 39 industrias españolas.

Otras medidas de concentración también de interés además de la longitud de la curva de Lorenz o de la familia introducida por Atkinson, A.B. (1970) aparte de las consideradas anteriormente son los Indices U introducidos y analizados por Davies, S. (1980) o el Coeficiente $\beta$ al que hace referencia Lafuente, A. y Salas, V. (1983). Este último ha sido empleado en el análisis de la concentración de las industrias americana e inglesa por varios autores, entre los que se encuentra Ijiri, Y. y Simon, H. (1971), Simon, H. y Bonini, C. (1958) y Hart, P.E. y Prais, S.J. (1956). Maravall, F. (1976) lo emplea para determinar la concentración de la industria española.

\section{Otras formas de medir la concentración industrial}

A partir de la década de los 60, numerosos autores han utilizado para medir la concentración distintas medidas relacionadas con la Teoría de la Información. El interés que despiertan las medidas relacionadas con la Teoría de la Información radica en la verificación de 
ciertas propiedades que permiten utilizarlas como indicadores para cuantificar el grado de concentración de un determinado mercado, o en sentido inverso, la incertidumbre asociada al mismo. Cabe resaltar la propiedad de descomponibilidad que poseen los indicadores que proceden de la Teoría de la Información, atrayendo a los especialistas por su significado y operatividad en el análisis de la concentración. Esta propiedad nos permite estudiar la concentración a diferentes niveles de agregación; esto es, podemos estudiar la concentración bien de cada uno de los sectores que componen la industria o bien en su conjunto pudiendo relacionar las concentraciones individuales con la agregada de forma que será posible conocer la influencia de cada uno de los sectores sobre el conjunto de la industria. En esta línea se encuentran los trabajos de Theil, H. (1967), Finkelstein, M.O. y Friedberg, R.M. (1967), Stigler, G. (1968), Horowitz, A. y Horowitz, I. (1968), Horowitz, I. (1970), Hexter, J.L. y Snow, J.W. (1970), Bourguignon, F. (1979), Shorrocks, A.F. (1980), Río, M.J. y Pérez, R. (1986, 1987, 1988, 1991), Eraso, L. y García, C. (1990) y Eraso, L. (1994) entre otros. Algunos de estos índices son:

a) Indice de Theil: Medida basada en la entropía de Shannon (Shannon, C.E. (1948)) e introducida por Theil, H. (1967) en este contexto, y por tanto debe ser interpretada como una medida inversa de concentración; es decir, una industria será más competitiva cuanto mayor sea la incertidumbre de que un determinado número de establecimientos o empresas puedan asegurarse un cliente al azar. Este índice viene dado por la siguiente expresión:

$$
E=-\sum_{i=1}^{N} p_{i} \log p_{i}
$$

En este caso la ponderación que se asigna a las empresas pequeñas es mayor que en el índice de Herfindahl como pone de manifiesto Miller, R.A. (1972). El valor máximo de E se alcanza cuando todas las empresas son de igual tamaño, siendo además $\mathrm{E}$ el logaritmo de su propio número equivalente. Horowitz, I. (1970) utiliza este procedimiento para analizar las similitudes existentes en la estructura industrial de Alemania, Francia, Italia, Holanda, Bélgica y Luxemburgo. Por último, indicar que el índice de Hannah-Kay se aproxima a éste cuando $\alpha$ se acerca a 1 . 
b) Indice de Gini-Simpson: Una generalización importante de las medidas de entropía son las de tipo $\beta$, introducidas por Havrda, J. y Charvat, F. (1967); y dentro de éstas se encuentra la entropía cuadrática para $\beta=2$, que salvo constante coincide con el índice cuadrático o índice de Gini-Simpson que viene dado por:

$$
I^{2}=1-\sum_{i=1}^{N} p_{i}^{2}
$$

Este índice fue introducido por Gini, C. (1912) y adaptado por Simpson, F.H. (1949) para medir la diversidad en una comunidad ecológica. Río, M.J. y Pérez, R. (1986, 1987, 1988, 1991) comprueban cómo este índice verifica ciertas propiedades básicas, que le permiten ser interpretado como indicador de la concentración industrial. Además de presentar un buen comportamiento frente a las axiomáticas de Hall-Tideman y Hannah-Kay este índice distingue más que el de Theil como ponen de manifiesto también estos autores. Otro trabajo en el que se incluye una amplia bibliografía en relación a este índice es el de Giorgi, G.M. (1993).

En aquellas situaciones donde no se dispone de la distribución completa del mercado surgen básicamente tres alternativas para abordar el problema, como son:

1) Completar la distribución con una empresa ficticia cuya participación sea la no asumida por las restantes.

2) Transformar las participaciones de cada empresa, de forma que la participación relativa total sea la unidad.

3) Abordar directamente el problema.

Las dos primeras alternativas pueden ser resueltas, evidentemente, a partir de la utilización de los diferentes índices analizados hasta el momento. En la línea de abordar directamente el problema se encuentra el trabajo de Eraso, L. y García, C. (1990) en el que se propone una familia de medidas en base a la entropía de Renyi (Renyi, A. (1961)). En este trabajo tras demostrar algunas propiedades se prueba que la familia introducida verifica las axiomáticas de Hall-Tideman y Hannah-Kay. En Eraso, L. (1994) se compara esta alternativa con la primera para este mismo índice.

Basadas en algunas de estas medidas de entropía se pueden definir diversas medidas de desigualdad. Así, por ejemplo, se tiene: 
c) Indice de desigualdad de Theil: Indice introducido por Theil, H. (1967) y ampliamente estudiado por Foster, J.E. (1983) viene dado por:

$$
I_{T h}=\log N+\sum_{i=1}^{N} p_{i} \log p_{i}
$$

y se encuentra englobado como caso particular de una familia más amplia analizada por Shorrocks, A.F. (1980).

En esta línea también se encuentra el Coeficiente de Variación que viene dado como la relación existente entre la desviación típica del tamaño de la empresa y el tamaño medio de la misma y cuya importancia radica en ser independiente de la unidad de medición. Esta y otras medidas derivadas de ella además de algunas de las consideradas anteriormente pueden verse en Egghe, L. y Rousseau, R. (1990, 1991).

Siguiendo en la línea de considerar medidas relacionadas con la Teoría de la Información y una vez revisadas brevemente algunas de las medidas más utilizadas procedentes de este contexto, se aborda el estudio de la familia de Entropías de Orden $\mathrm{r}$ introducida por Renyi (1961) como medidas que permiten cuantificar la incertidumbre o falta de certeza que se tiene acerca de una determinada situación proporcionando una valoración del grado de competencia entre las empresas de un determinado sector y, por tanto, en sentido opuesto indicarán su nivel de concentración. El buen comportamiento de esta familia con respecto a las diferentes axiomáticas planteadas constituye una base sólida para su utilización como medida de concentración industrial, bien directamente como puede verse en Mayoral, M. (1995) o bien considerando alguna modificación como plantean Eraso, L. y García, C. (1990).

Esta familia surge como un primer intento para llevar a cabo una generalización de la entropía de Shannon, dada por Shannon (1948) y que puede considerarse como la primer medida que permite cuantificar la incertidumbre asociada a un experimento aleatorio. Aunque, históricamente el primero en tratar el problema fue Hartley (1928) quien propuso como medida de la incertidumbre asociada a una experiencia que puede dar lugar a $\mathrm{N}$ resultados diferentes, el logaritmo de dicho número de resultados.

$$
-194-
$$


Este primer intento de generalización llevado a cabo por Renyi (1961), viene dado en los siguientes términos:

«Sea $X$ una variable o proceso de clasificación que divide a la población en $N$ clases o categorías, $x_{1}, \ldots, x_{N}$, que se presentan con frecuencias relativas $p_{1}, \ldots, p_{N}\left(\mathrm{p}_{1} \geqslant 0, \ldots, p_{N} \geqslant 0, \sum_{i=1}^{N} p_{i}=1\right)$. Se denomina Entropía de Orden $\mathrm{r}$ de Renyi de X al valor de la expresión

$$
H(P)=H\left(p_{1}, \ldots, p\right)=\left\{\begin{array}{lll}
1 / 1-r \log \left(\sum_{i=1}^{N} p_{i}\right) & \text { si } & r \neq 1, r>0 \\
-\sum_{i=1}^{N} p_{i} \log p_{i} & \text { si } & r=1
\end{array}\right.
$$

si denotamos $P=\left(p_{1}, \ldots, p_{N}\right)$ y definimos $p_{i}=0$ y $p_{i} \log p_{i}=0$ cuando $p_{i}=0$, donde los logaritmos se pueden tomar con respecto a cualquier base»

Esta familia contiene como caso particular a la medida de Shannon para $r=1$ y cuando $r$ tiende a 0 se obtiene la medida dada por Hartley. Además, en cuanto que puede interpretarse como una medida de la incertidumbre relativa al resultado de un experimento $\mathrm{X}$, se puede considerar como una valoración del grado de competencia entre los establecimientos que integran la industria; luego, en sentido opuesto, representará el grado de concentración de la misma. Evidentemente, esta familia verifica los axiomas 1 y 2 de Hall y Tideman.

Algunas de las propiedades más importantes que verifican las medidas de entropía y en particular la Entropía de Orden r, que permiten interpretar a los elementos de esta familia como buenos indicadores de la concentración industrial, se recogen a continuación:

1) Es siempre no negativa.

2) No se modifica por la inclusión de una categoría con frecuencia relativa nula

3) Si todas las frecuencias relativas son iguales entonces es función del número de categorías distintas $\mathrm{N}$ y crece al crecer éste.

4) Es una función decreciente de $r$ para $\left(p_{1}, \ldots ., p_{N}\right)$ fijo.

5) Alcanza un máximo cuando todas las categorías tienen la misma frecuencia. 
6) Alcanza el valor mínimo si existe una categoría con frecuencia uno.

7) Es una función simétrica de sus argumentos.

8) Es una función continua de sus argumentos.

9) (Propiedad de Ramificación)

$$
H_{N}^{r}\left(p, \ldots, p_{N}\right)=H_{N-1}^{r}\left(p_{1}+p_{2}, p_{3}, \ldots, p_{N}\right)+\left(p_{1}+p_{2}\right) H_{2}^{r}\left(\frac{p_{1}}{p_{1}+p_{2}}, \frac{p_{2}}{p_{1}+p_{2}}\right)
$$

10) (Principio de transferencia)

Se considera un sistema de frecuencias $\left\{p_{1}, \ldots, p_{N}\right\}, p_{i} \geqslant 0, \mathrm{i}=1, \ldots, \mathrm{N}$, $\sum_{i=1}^{N} p_{i}=1$, en el que suponemos que $p_{1}>p_{2}$. Si se construye un nuevo sistema de frecuencias $\left\{q_{1}, \ldots ., q_{N}\right\}$ en el cual $q_{1}=p_{1}-\varepsilon, q_{2}=p_{2}+\varepsilon, q_{3}$ $=p_{3}, \ldots ., q_{N}=p_{N}$ con $0<\varepsilon<\left(p_{1}-p_{2}\right) / 2$, entonces

$$
H_{N}^{r}\left(q_{1}, \ldots ., q_{N}\right) \geqslant H_{N}^{r}\left(p_{1}, \ldots ., p_{N}\right)
$$

A partir de estas propiedades se tiene que la incertidumbre acerca del resultado de un experimento es siempre positiva (propiedad 1), lo cual en términos de concentración nos indica que la concentración asociada a una determinada distribución de mercado es siempre positiva. Además (propiedad 2) la incertidumbre se conserva al considerar una nueva categoría que nunca puede darse. Esto es, la inclusión en el mercado de un establecimiento o empresa con participación nula no influye en la concentración. Sobre la base de este resultado, se puede suponer sin pérdida de generalidad que los establecimientos o empresas que integran el estudio tienen participación positiva ya que, de lo contrario, su influencia en la concentración es nula.

Así, por ejemplo, si consideramos 6 empresas que integran un determinado sector cuyas cuotas de mercado se supone que vienen dadas por las frecuencias relativas, $p_{1}=.2, p_{2}=.1, p_{3}=.1, p_{4}=.3, p_{5}=.2$ y $p_{6}=.1$, la Entropía de Orden $r$, para $\mathrm{r}=2$, viene dada por

$$
\begin{gathered}
H_{6}^{2}(.2, .1, .1, .3, .2, .1)=1.609438 . \\
-196-
\end{gathered}
$$


De la propiedad 3 se desprende que si un sector industrial está formado por $\mathrm{N}$ establecimientos o empresas de igual tamaño la concentración decrece en función de $\mathrm{N}$ ya que $\mathrm{H}$ es una función creciente de N. Este resultado no es más que el axioma 5 de Hall y Tideman.

La propiedad 4 nos permite obtener una cota superior para la Entropía de Orden $r$ que viene recogida en la propiedad 5 y que nos indica que la mayor incertidumbre sobre qué categoría se presenta cuando las frecuencias de todas las clases coinciden. Por tanto, esta situación se corresponde con el caso de competencia perfecta, la participación total está repartida de forma uniforme lo que nos lleva a la existencia de una nula concentración.

Si evaluamos ahora la Entropía de Orden $r$, para $r=4$, en el caso anterior se obtiene que

$$
H_{6}^{4}(.2, .1, .1, .3, .2, .1)=1.485583
$$

Si la situación de partida cambia y la distribución de mercado pasa a ser $p_{1}=.4, p_{2}=.1, p_{3}=.1, p_{4}=.2, p_{5}=.1$ y $p_{6}=.1$, la Entropía de Orden $r$, para $r=2$, viene dada por

$$
H_{6}^{2}(.4, .1, .1, .2, .1, .1)=1.427116
$$

mientras que si $r=4$

$$
H_{6}^{4}(.4, .1, .1, .2, .1, .1)=1.196647 \text {. }
$$

Por tanto, al aumentar $\mathrm{r}$ y pasar de una situación de menor a mayor concentración el índice detecta mejor esa concentración al ponderar más las categorías con frecuencias mayores.

Como el máximo de esta función depende del número de categorías, podemos estandarizar la Entropía de Orden $\mathrm{r}$ para que su valor máximo sea 1, como plantean Eraso, L. y García, C. (1990).

La propiedad 6 plantea la situación en la cual la incertidumbre es nula. En esta situación, como la participación corresponde a un único establecimiento (una única empresa) nos encontramos ante una situación de monopolio, es decir de máxima concentración. 
En nuestro ejemplo, como el número de categorías es 6 , la medida varía entre 0 (caso de máxima concentración) y 1.791759 (caso de nula concentración).

La propiedad 7 permite calcular la concentración de modo imparcial y manteniendo en el anonimato a los establecimientos o empresas que participan en el sector ya que este índice no depende del orden en que se consideren los valores o clases, es decir

$$
H_{6}^{2}(.2, .1, .1, .3, .2, .1)=1.609438=H_{6}^{2}(.1, .3, .1, .2, .2, .1)
$$

La propiedad 8 garantiza el que pequeñas variaciones en los tamaños relativos de las empresas llevan asociados una pequeña modificación en la concentración del sector (Axioma 6 de Hannah y Kay).

Como consecuencia inmediata de la propiedad 9 se tiene que

$$
H_{N}^{r}\left(p_{1}, \ldots, p_{N}\right) \geqslant H_{N-1}^{r}\left(p_{1}+p_{2}, p_{3}, \ldots, p_{N}\right) .
$$

lo que nos indica que la incertidumbre disminuye al fusionarse dos empresas con participación en el mercado $p_{1}$ y $p_{2}$ dando lugar a un nuevo establecimiento (una nueva empresa) con participación $p_{1}+p_{2}$ existiendo, por tanto, mayor concentración. Esto implica el axioma 4 de Hall y Tideman, en cuanto a que conlleva la idea de aumento de la concentración cuando se produce alguna fusión, así como el axioma 4 de Hannah y Kay.

Así, si se produce una fusión entre las dos primeras empresas y la distribución de mercado inicial, $p_{1}=.2, p_{2}=.1, p_{3}=.1, p_{4}=.3, p_{5}=.2 \mathrm{y}$ $p_{6}=.1$, pasa a ser $p_{1}+p_{2}=.3, p_{3}=.1, p_{4}=.3, p_{5}=.2$ y $p_{6}=.1$ se tiene que, para $\mathrm{r}=2$,

$$
H_{6}^{2}(.2, .1, .1, .3, .2, .1)=H_{5}^{2}(.3, .1, .3, .2, .1)+.3 H_{2}^{2}(.2 / .3, .1 / .3) .
$$

También de esta propiedad se tiene la descomponibilidad del indicador propuesto lo que constituye la principal cualidad, de este tipo de medidas, que atrajo la atención de los especialistas en cuanto a su significado y operatividad en el análisis de la concentración. Esta propiedad es especialmente deseable cuando se trabaja con poblaciones estratificadas. En este caso, es posible determinar la concentración poblacional como suma de la concentración entre los estratos y 
un promedio de la concentración dentro de los estratos. Lo cual, permite hacer comparaciones entre los distintos estratos en los que es posible dividir la población.

Así, si suponemos que la población está formada por L estratos, la concentración global se puede obtener en los siguientes términos (Gil, P. 1981):

$$
\begin{gathered}
H_{M}^{r}\left(q_{11}, \ldots, q_{1 M_{1}}, \ldots, q_{L 1}, \ldots, q_{L M_{L}}\right)=H_{M}^{r}\left(p_{1}, \ldots, p_{L}\right)+ \\
+\sum_{K=1}^{L} p_{k} H_{M}^{r}\left(\frac{q_{k 1}}{p_{k}}, \ldots, \frac{p_{k M_{k}}}{p_{k}}\right)
\end{gathered}
$$

donde $H_{M}^{r}\left(q_{11}, \ldots, q_{1 M}, \ldots, q_{L M}, \ldots, q_{L M_{L}}\right)$ representa la concentración global, $H_{M}^{r}\left(p_{1}, \ldots, p_{L}\right)$ la concentración entre estratos y $H_{M}^{r}\left(\frac{q_{k 1}}{p_{k}}, \ldots, \frac{q_{k} M_{k}}{p k}\right)$ la concentración dentro del estrato $\mathrm{k}$, siendo $p_{k}=\sum_{i=1}^{M k} q_{k i}$ para todo $\mathrm{k}=1$,

Por último, la propiedad 10 reafirma el comportamiento de la Entropía de Orden $r$ como una medida de incertidumbre, indicando que un cambio en las frecuencias relativas en el sentido de igualarlas produce un incremento de la incertidumbre. Luego, si un establecimiento (empresa) pierde parte de su cuota de mercado en favor de otro más pequeño, se producirá una disminución en la concentración del sector (Axioma 3 de Hall y Tideman y axioma 2 de Hannah y Kay). Además de la aplicación reiterada de este principio de transferencia se obtienen los axiomas 1 y 3 de Hannah y Kay.

Todas estas propiedades ponen de manifiesto el buen comportamiento de las Entropías de Orden $r$ en relación a las dos axiomáticas consideradas, lo que permite interpretarlas como buenos indicadores de la concentración de un determinado mercado, ya que cuantifican la incertidumbre asociada a la distribución del mismo.

Así, cuanto mayor sea el valor del índice, mayor es el grado de incertidumbre respecto a cuál de los establecimientos o empresas asumirá la demanda de un determinado cliente elegido al azar. Es decir, proporciona una valoración del grado de competencia entre los establecimientos o empresas del mercado analizado y por tanto, indicará el grado de concentración del mismo. De forma que, a mayor incertidumbre existirá menor concentración y a medida que aquella decrece la concentración aumentará.

$$
-199-
$$




\section{BIBLIOGRAFIA}

AulLo, E. (1979): «El comportamiento teórico de los índices de concentración: Un ejercicio de aplicación a la industria española». Cuadernos de Economía, 7, 18, 3-27.

Atkinson, A. B. (1970): «On the measurement of inequality». Journal of Economic Theory, 2, 244-263 (Citado en Davis (1980)).

BAyle, D. y Boyle, S. E. (1971): «The optimal measure of concentration». Journal of the American Statistical Association, 66, 336, 702-706.

Bourguignon, F. (1979): «Descomposable income inequality measures». Econométrica, 47, 901-920 (Citado en Foster (1983)).

Clarke, R. (1993): Economía Industrial. Celeste Ediciones. (Traducción de P. L. Marín Urive y A. Sanchís).

Curry, B. y George, K. D. (1983): «Industrial Concentration: a survey». Journal of Industrial Economic, 31, 203-255.

Dalton, H. (1920): «The measurement of the inequality of incomes». The Economic Journal, 30, 348-361 (Citado en Foster (1983)).

DAviES, S. (1980): «Measuring industrial concentration: an alternative approach». Review of Economics and Statistics, 62, 2, 306-309.

EGGHe, L. y RousseaU, R. (1990): «Elements of concentration theory». In L. Egghe \& R. Rousseau (Eds.), Informetrics 89/90, Elsevier Science Publishers, 97-137.

EGGHe, L. y Rousseau, R. (1991): «Transfer principles and a classification of concentration measures». Journal of the American Society for Information Science, 42, 7, 479489.

Eraso, L. y Garcia, C. (1990): «Concentración industrial y medidas de información». Investigaciones Económicas, XIV, 2, 225-237.

ERASo, L. (1994): «Sobre la elección del índice de información para distribuciones in completas». Actas del XXI Congreso Nacional de Estadística e Investigación Operativa, 407-408.

Finkelstein, M. O. y Friedberg, R. M. (1967): «The application of an entropy theory of concentration to the Clayton Act». The Yale Law Review, 76, 677-717 (Citado en Horowitz (1970)).

Foster, J. E. (1983): «An axiomatic characterization of the Theil measure of income inequality». Journal of Economic Theory, 31, 105-121.

GIL, P. (1981): Teoría matemática de la Información. I.C.E (1. ${ }^{\mathrm{a}}$ edición).

GiNI, C. (1912): Variabilita e Mutabilita. Studi Economicoaguridici della Facotta di Giurisprudenza dell Universite di Cagliari III. Parte II (Citado en Mathai y Rathie (1975)).

GioRGI, G. M. (1993): «A fresh look at the topical interest of the Gini concentration ratio». Metron, LI, 1-2, 83-98.

González, J. L. (1988): «Crecimiento y tamaño de la empresa. Una aplicación al sector textil catalán». Boletín de Estudios Económicos, XLIII, 135, 503-520.

Hall, M. y Tideman, N. (1967): «Measures of concentration». Journal of American Statistical Association, 62, 162-168 (Citado en Curry y George (1983)).

HANNAH, L. y KAY, J. A. (1977): Concentration in modern industry: theory, measurements and the U. K. experience. Macmillan (Citado en Curry y George (1983)).

Hartley, R. (1928): «Transmission of information». The Bell System Technical Journal, 7, 535-563 (Citado en Mathai y Rathie (1975)).

HART, P. E. y PraIs, S. J. (1956): «The analysis of business concentration: a statistical approach». Journal of the Royal Statistical Society. Serie A, 119, 2, 150-191 (Citado en Lafuente y Salas (1983)).

HAUSE, J. C. (1977): «The measurement of concentrated industrial structure and the size distribution of firms». Annals of Economic and social measurement, 6, 1 (Citado en Aguiló (1979)). 
Havrda, J. y ChaRvat, F. (1967): «Quantification method of classification processes». Kybernetika, 3, 30-35.

Herfindahl, O. C. (1950): Concentration in the steel industry. Unpublished Ph. D. dissertation, Columbia University (Citado en Clarke (1993)).

Hexter, J. L. y SNow, J. W. (1970): «An entropy measure of relative aggregate concentration». Southern Economic Journal, 36, 239-243.

Hirschman, A. O. (1945): National power and the structure of foreign trade. Berkeley and Los Angeles: University of California Press (Citado en Clarke (1993)).

Hirschman, A. O. (1964): "The paternity of an index». American Economic Review, 54, 761-762 (Citado en Clarke (1993)).

Horowitz, A. y Horowitz, I. (1968): «Entropy, markov processes and competition in the brewing industry». Journal of Industrial Economics, XVI, 196-211 (Citado en Horowitz (1970)).

Horowitz, I. (1970): «Employment concentration in the common market: an entropy approach». Journal of Industrial Economics, Journal of the Royal Statisical Society, Serie A, 133, 463-479.

Horvath, J. (1970): «Suggestion for a comprehensive measure of concentration». Southern Economic Journal (Citado en Aguiló (1979)).

IJIRI, Y. y Simon, H. (1971): «Effects of mergers and acquisitions on business firm concentration». Journal of Political Economy, 79 (Citado en Lafuente y Salas (1983)).

LAfuente, A. y SAlas, V. (1983): «Concentración y resultados de las empresas en la economía española». Cuadernos Económicos del I.C.E., 22-23, 6-20.

Maravall, F. (1976): Crecimiento, dimensión y concentración de las empresas industriales españolas. Fundación del I.N.I. Serie E, n. ${ }^{\circ} 7$ (Citado en Lafuente y Salas (1983))

MARFELS, C. (1971): «Absolute and relative measures of concentration». Kyklos, 24, 4, 753-766 (Citado en Aguiló (1979)).

Mathai; A. N. y RAthie, P. N. (1975): Basic concepts in Information Theory and Statistics. Wiley Eastern Limited. New Delhi.

Mayoral, M. M. (1995): Una aproximación a la realidad industrial en la Comunidad de Madrid a través de la Entropia de Orden $r$. Ph. D. Thesis, Universidad Complutense de Madrid.

MilleR, R. A. (1971): «Marginal concentration ratios as market structure variables». Review of Economics and Statistics, 53, 289-293 (Citado en Aguiló (1979)).

Miller, R. A. (1972): «Numbers equivalents, relative entropy and concentration ratios; a comparisons using market performance». Shothern Economic Journal.

Pigou, A. C. (1912): Wealth and welfare. Macmillan (Citado en Foster (1983)).

Renyi, A. (1961): "On the measures of entropy and information». Proc. 4th. Berkeley Symp. Math. Statist. and Prob., 1, 547-561 (Mathai, A.N. y Rathie, P.N. (1975)).

Río, M. J. y PÉREz, R. (1986): «Grado de concentración industrial: una aproximación». Actas XVI Reunión Nacional de la SEIO.

Río, M. J. y PÉREz, R. (1987): «El índice cuadrático como medida de la concentración industrial». XII Jornadas Luso-Españolas de Matemáticas. Braga (Portugal).

Río, M. J. y PÉrez, R. (1988): «Sobre la medición de la concentración industrial». Investigaciones Económicas. Suplemento, 81-88.

Rí, M. J. y Pérez, R. (1991): Cuantificación de la concentración industrial: un enfoque analítico. Documentos de Trabajo. Facultad de C.C. Económicas y Empresariales. Universidad de Oviedo.

Shannon, C. E. (1948): «A mathematical theory of comunication». The Bell System Technical Journal, 27, 379-423 (Citado en Gil (1981)).

SHorrocks, A. F. (1980): «The class of additively descomposable inequality measures». Econometrica, 48, 3, 613-625 (Citado en Foster (1983)). 
Simon, H, y Bonini, C. (1958): «The size distributiom of business firms». American Economic Review, 48, 607-617 (Lafuente, A. y Salas, V. (1983)).

Simpson, F. H. (1949): «Measurement of diversity». Nature, 163, 688.

STIGLER, G. J. (1968): The organization of industry. Homewood. Illinois: Irwin $\left(1 .^{\text {a }}\right.$ edición).

TheIL, H. (1967): Economics and Information Theory. North-Holland (1. a edición).

RESUMEN: En este trabajo se lleva a cabo una revisión de la concentración industrial desde el punto de vista de su medición y de la problemática que ello plantea. Este concepto así como su cuantificación es un tema con importantes aplicaciones en diferentes campos de investigación entre los que se encuentra la Geografía Industrial. 